\title{
Factores morfológicos asociados al pronóstico de pacientes operados por cáncer gástrico incipiente*
}

\author{
Drs. ÓSCAR TAPIA E..$^{1,2}$, PATRICIA GARCÍA M. ${ }^{2}$, CARLOS MANTEROLA D. ${ }^{3}$, \\ MIGUEL VILLASECA H. ${ }^{1,2}$, JUAN CARLOS ARAYA O. ${ }^{1,2}$, JUAN CARLOS ROA S..$^{1,2}$ \\ 1 Departamento de Anatomía Patológica. Facultad de Medicina. \\ 2 Scientifical and Technological Bioresources Nucleus (BIOREN). \\ 3 Departamento de Cirugía y Traumatología, Facultad de Medicina. \\ Universidad de La Frontera, Temuco, Chile.
}

\begin{abstract}
Pathological features associated with survival in early gastric cancer

Background: Early gastric cancer corresponds to those tumors that only involve mucosa and submucosa. It is associated with a high survival rate. Aim: To determine pathological factors associated with survival in early gastric cancer. Material and Methods: Analysis of pathological records of 106 patients, with a median age of 63 years $(60 \%$ males), subjected to a gastrectomy for early gastric cancer. Follow up was performed according to data in the clinical records and death certificates obtained at the Chilean National Death Registry. Results: Five years global survival of patients was $91 \%$. Lymph node involvement was more common among tumors bigger than $35 \mathrm{~mm}$, with a low degree of differentiation and among those tumors classified as diffuse according to Lauren. Survival was significantly lower for bigger tumors, those with of a low degree of differentiation, diffuse tumors according to Lauren and those with lymph node involvement. Conclusions: Early gastric cancer has a high five years survival. Bigger tumors, those with a low degree of differentiation and those with lymph node involvement are associated with lower survival rates.
\end{abstract}

Key words: Early gastric cancer, survival, prognosis.

\section{Resumen}

Introducción: El cáncer gástrico incipiente (CGI) es aquel que compromete la mucosa o submucosa gástrica independientemente del compromiso ganglionar linfático, estimándose su prevalencia en Chile inferior al $20 \%$. El objetivo de este estudio es determinar prevalencia de CGI y asociación de variables biodemográficas y morfológicas con la supervivencia (SV) de pacientes resecados por CGI. Material y Método: Estudio de cohorte retrospectiva. Se estudiaron variables biodemográficas y morfológicas de 106 pacientes resecados por CGI entre 1986-2007. Se aplicó estadística descriptiva y analítica; confección de curvas de $\mathrm{SV}$, y finalmente se aplicaron modelos de regresión logística para realizar ajuste, calcular odds ratio y sus respectivos intervalos de confianza de $95 \%$. Resultados: $15 \%$ correspondió a CGI. La mediana de edad fue

*Recibido el 14 de Julio de 2010 y aceptado para publicación el 3 de Agosto de 2010.

Financiado en parte por la Dirección de investigación de la Universidad de La Frontera. DI07-0111

Correspondencia: Dr. Óscar Tapia E.

Manuel Montt 112. Código Postal 478-1176, Temuco, Chile.

otescalona@gmail.com 
FACTORES MORFOLÓGICOS ASOCIADOS AL PRONÓSTICO DE PACIENTES OPERADOS POR CÁNCER GÁSTRICO...

63 años y el $60 \%$ correspondió a género masculino con una SV global a 5 años de $91 \%$. Se observaron diferencias estadísticas significativas entre tumores mucosos y submucosos en cuanto a la localización tumoral y compromiso linfonodal junto con presentarse el compromiso nodal más frecuentemente en tumores $\geq 35 \mathrm{~mm}$ poco diferenciados y difusos de Lauren. El análisis multivariado identificó como factores asociados a la SV: tamaño tumoral, grado de diferenciación histológica en su variedad poco diferenciado, tipo difuso de Lauren y compromiso ganglionar linfático. Conclusiones: Se verificó una prevalencia de CGI de 15\%, los que resecados presentan SV de $91 \%$ a 5 años. El compromiso linfonodal es un factor asociado a la SV; y además, se relaciona con tamaño tumoral, tipo histológico según Lauren, grado de diferenciación histológico y nivel de infiltración.

Palabras clave: Cáncer gástrico incipiente, pronóstico, factores de riesgo.

\section{Introducción}

El cáncer gástrico incipiente (CGI), fue definido en 1962 por la Sociedad Japonesa para estudio del cáncer gástrico $(\mathrm{CG})$, para referirse a aquellos tumores que invaden la mucosa o submucosa gástrica, independientemente del tamaño y compromiso ganglionar linfático ${ }^{1}$. La prevalencia del CGI es ampliamente variable dependiendo de la población estudiada, así en Japón, corresponde aproximadamente a un $50 \%$ de los $\mathrm{CG}$ diagnosticados mientras que estudios nacionales han reportado cifras que no superan el $20 \% 0^{2-5}$. Por esta razón, en nuestro país se han creado políticas de salud destinadas a efectuar un diagnóstico precoz en población de alto riesgo con tratamiento en tiempos garantizados y de esta forma lograr reducir las tasas de morbimortalidad por $\mathrm{CG}$, pues en estadios precoces se ha reportado que la supervivencia (SV) global a 5 años es de hasta $92 \%{ }^{6}$.

Dado el buen pronóstico observado en este subgrupo de pacientes con $\mathrm{CG}$, se ha llegado a recomendar la realización de procedimientos menos invasivos con la finalidad de tener menor impacto en la calidad de vida de estos pacientes; estos incluyen la resección mucosa y disección submucosa de tumores incipientes por vía endoscópica, inicialmente desarrolladas como un procedimiento diagnóstico, pero con el tiempo perfeccionadas y consideradas como herramientas terapéuticas para tumores pequeños (de diámetro menor a $1 \mathrm{~cm}$ ) y lesiones menores de $3,5 \mathrm{~cm}$ que no presentan invasión vascular ni permeaciones tumorales linfáticas ${ }^{7-11}$.

Por otra parte, se ha reportado invasión ganglionar linfática hasta en el 3\% de los CG intramucosos y $20 \%$ en CG submucoso, con un promedio de $14 \%$ para el grupo de los CGI; encontrándose ampliamente documentada su importancia pronóstica tanto en la recurrencia como SV de estos pacientes, y existiendo a su vez una asociación entre el estado ganglionar linfático con la localización y tamaño tumoral, el grado de diferenciación histológico, el nivel de infiltración y la presencia de permeaciones tumorales vasculares. Es por ello que se recomienda realizar gastrectomía con disección ganglionar en tumores incipientes sobre los $3,5 \mathrm{~cm}$. de diámetro mayor, en aquellas lesiones poco diferenciadas y con permeaciones tumorales vasculares, de forma tal de ofrecer una "cirugía con intención curativa" a este grupo de pacientes ${ }^{7-10}$.

El objetivo de este estudio fue determinar prevalencia de CGI y existencia de asociación de variables biodemográficas y morfológicas con la SV de pacientes resecados por CGI.

\section{Material y Método}

Diseño del estudio: Estudio de cohorte histórica o retrospectiva.

Participantes: Se recogieron los datos provenientes de pacientes diagnosticados de CGI en la Unidad de Anatomía Patológica del Hospital Hernán Henríquez Aravena de la ciudad de Temuco entre enero de 1986 y diciembre de 2007, incluyéndose todos los casos de gastrectomía por CGI diagnosticados de forma consecutiva. En todos los pacientes se realizó gastrectomía radical incluyendo omentectomía y linfadenectomía D2 (grupos ganglionares 1 al 9). El seguimiento se realizó mediante el estudio de las historias clínicas y certificados de defunción obtenidos del Registro Civil e Identificación.

Protocolo de procesamiento. En este período, el procesamiento de las piezas quirúrgicas fue realizado de forma uniforme a través de la apertura, extensión y fijación de la pieza en planchas de parafina sólida, con examen macroscópico diferido. Todas las lesiones sospechosas de cáncer reconocidas al examen macroscópico, fueron muestreadas en su totalidad con el objeto de determinar el máximo nivel de infiltración de la lesión, considerando también el examen microscópico del estudio de ganglios linfáticos. En todos los casos se realizó examen histopatológico diferido de ambos bordes de sección quirúrgicos (proximal y distal) incluyendo además el estudio con biopsia intraoperatoria del borde proximal y/o distal según la localización tumoral.

Variables de interés: La variable resultado fue $\mathrm{SV}$, medida en meses posterior a la cirugía. Las 
variables de control fueron: biodemográficas (género y edad) y morfológicas (localización, tamaño tumoral, tipo macroscópico, grado de diferenciación histológico, tipo histológico según Lauren, nivel de infiltración tumoral, compromiso y estadio ganglionar linfático).

Plan de análisis: Utilizando los paquetes estadísticos Epi-info 6.0 y Stata 9.0, se realizó un análisis exploratorio de los datos, lo que determinó la necesidad de categorizar algunas variables según su distribución: tamaño tumoral (dicotomizada en menor e igual o mayor de $35 \mathrm{~mm}$ ), localización macroscópica de la lesión (categorizada en tercios superior o cardial, medio e inferior o antral; según clasificación japonesa para estudio del CG), tipo macroscópico (categorizada según la clasificación macroscópica de la sociedad japonesa de gastroenterología endoscópica para tumores incipientes en tipo I [elevado], II [plano] y III [excavado]), grado de diferenciación histológica (categorizado en bien diferenciado, moderadamente diferenciado y poco diferenciado; prevaleciendo para su categorización el menor grado de diferenciación presente en el tumor), tipo histológico según Lauren (categorizado en variante intestinal y difuso en base a las definiciones ya establecidas en la literatura), nivel de infiltración (categorizado según el compromiso de la pared gástrica en tumores intramucosos y submucosos); número de ganglios linfáticos resecados (considerándose para ello el número total de linfonodos examinados), compromiso ganglionar linfático (dicotomizado en positivos [comprometidos por tumor] o negativos [no comprometidos por tumor]) y estadio ganglionar linfático (de acuerdo a la definición de la $7^{\circ}$ edición AJCC para compromiso ganglionar linfático en N0, N1, N2 y N3).

Sesgos: Se minimizó el sesgo de clasificación mediante la comprobación de los datos por investigadores independientes con amplia experiencia en el tema (JCR, MV y JCA).

Tamaño de la muestra: No se realizó estimación de tamaño de la muestra pues se trabajó con la totalidad del universo de pacientes con CGI en el período señalado.

Métodos estadísticos: Se aplicó estadística descriptiva con cálculo de medidas de tendencia central y extrema; y estadística analítica, aplicando Chi cuadrado de Pearson y exacto de Fisher para comparación de variables categóricas, T-student para comparación de promedios en aquellas variables continuas; confección de curvas de Kaplan-Meier y aplicación de Log-rank test para análisis de SV. Finalmente, se aplicaron modelos de regresión logística para determinación de fuerza de asociación con cálculo de odds ratio (OR) y sus respectivos intervalos de confianza de 95\% (IC 95\%).

\section{Resultados}

En el período antes señalado se realizaron 694 gastrectomías; de los cuales se verificaron 106 casos de CGI; por lo que se reporta una prevalencia de CGI de $15 \%$.

La mediana de edad de los casos de CGI fue de 63 años y el $60 \%$ (64) de los pacientes eran de género masculino. El 44\% (47) y 56\% (59) correspondieron a tumores intramucosos y submucosos respectivamente; no demostrándose compromiso tumoral de los bordes proximal ni distal de sección en ninguno de los casos estudiados, tanto en el examen histopatológico intraoperatorio como diferido.

Al comparar ambos subgrupos sólo se constataron diferencias estadísticamente significativas en las variables localización del tumor (en tercio superior) y compromiso ganglionar linfático en lesiones submucosas. En cuanto al tipo histológico según Lauren, el 64\% correspondió a la variante intestinal (30/47 intramucosos y $37 / 59$ submucosos) y $36 \%$ difuso (17/47 intramucosos y 22/59 submucosos) $(\mathrm{p}=0,90)($ Tabla 1$)$.

Se observó compromiso ganglionar linfático en el 12\% del grupo total (13 casos) (Tabla 1). Según tamaño tumoral, se verificó compromiso ganglionar en el $6 \%$ (1/34 intramucosos y $3 / 32$ submucosos) para tumores $\leq 35 \mathrm{~mm}$ y en el $23 \%$ (1/13 intramucosos y $8 / 27$ submucosos) para tumores $>35 \mathrm{~mm}$ $(\mathrm{p}=0,01)$. Se verificó compromiso nodal en el $6 \%$ de aquellos tumores bien/moderadamente (1/29 intramucosos y $3 / 34$ submucosos), mientras que en tumores poco diferenciados se observó compromiso en el 21\% (1/18 intramucosos y 8/25 submucosos) $(\mathrm{p}=0,02)$. Para variante intestinal y difuso de Lauren se demostró compromiso nodal en el 4\% (0/30 intramucosos y $3 / 37$ submucosos) y $26 \%(2 / 17$ intramucosos y $8 / 22$ submucosos) respectivamente $(\mathrm{p}=0,001)$.

Con una mediana de seguimiento de 63 meses (7 a 187) la SV global actuarial observada a 5 y 10 años fue de $91 \%$ y $86 \%$ respectivamente.

Al aplicar análisis bivariados se constató asociación entre SV y las variables localización de la lesión $(\mathrm{p}=0,01)$, tamaño tumoral $(\mathrm{p}=0,03)$ (Figura $1)$, nivel de infiltración $(\mathrm{p}=0,02)$ (Figura 2$)$, grado de diferenciación histológica $(\mathrm{p}=0,01)$ (Figura 3), tipo histológico según Lauren $(\mathrm{p}=0,003)$ y compromiso ganglionar linfático $(\mathrm{p}<0,001)$ (Figura 4). El análisis multivariado permitió verificar como variables asociadas a la SV en pacientes con CGI al compromiso tumoral ganglionar linfático, al grado de diferenciación histológica en su variedad poco diferenciado, al tipo difuso de Lauren y al tamaño tumoral superior a $35 \mathrm{~mm}$ (Tabla 2). 
FACTORES MORFOLÓGICOS ASOCIADOS AL PRONÓSTICO DE PACIENTES OPERADOS POR CÁNCER GÁSTRICO...

Tabla 1. Distribución de variables biodemográficas y morfológicas según nivel de infiltración tumoral

\begin{tabular}{|c|c|c|c|}
\hline Variables & $\begin{array}{l}\text { Intramucoso } \\
(n=47)\end{array}$ & $\begin{array}{l}\text { Submucoso } \\
\quad(n=59)\end{array}$ & $\mathbf{p}$ \\
\hline Género \% (n) & & & 0,58 \\
\hline Femenino & $43,0(20)$ & $37,0(22)$ & \\
\hline Masculino & $57,0(27)$ & $63,0(37)$ & \\
\hline Edad (años) & $59,7 \pm 13,6$ & $62,7 \pm 11,1$ & 0,89 \\
\hline Resección quirúrgica (\%) & & & 0,19 \\
\hline Gastrectomía subtotal & $62,0(29)$ & $49,0(29)$ & \\
\hline Gastrectomía total & $38,0(18)$ & $51,0(30)$ & \\
\hline Localización (\%) & & & 0,005 \\
\hline $1 / 3$ superior & $19,0(9)$ & $39,0(23)$ & \\
\hline $1 / 3$ medio & $34,0(16)$ & $42,0(25)$ & \\
\hline $1 / 3$ inferior & $47,0(22)$ & $19,0(11)$ & \\
\hline Tipo macroscópico (\%) & & & 0,23 \\
\hline Tipo I & $13,0(6)$ & $26,0(15)$ & \\
\hline Tipo II & $79,0(37)$ & $64,0(38)$ & \\
\hline Tipo III & $8,0(4)$ & $10,0(6)$ & \\
\hline Tamaño tumoral & & & 0,05 \\
\hline$\leq 35 \mathrm{~mm}$ & $72,0(34)$ & $54,0(32)$ & \\
\hline$>35 \mathrm{~mm}$ & $28,0(13)$ & $46,0(27)$ & \\
\hline Número de ganglios resecados & $27,4 \pm 14,7$ & $24,6 \pm 12,6$ & 0,14 \\
\hline Estado ganglionar linfático (pN) & & & 0,02 \\
\hline pN0 & $96,0(45)$ & $81,0(48)$ & \\
\hline $\mathrm{pN} 1$ & $4,0(2)$ & $12,0(7)$ & \\
\hline $\mathrm{pN} 2$ & - & $7,0(4)$ & \\
\hline $\mathrm{pN} 3$ & - & - & \\
\hline Compromiso ganglionar linfático & & & 0,02 \\
\hline Negativo & $96,0(45)$ & $81,0(48)$ & \\
\hline Positivo & $4,0(2)$ & $19,0(11)$ & \\
\hline Tipo histológico según Lauren & & & 0,90 \\
\hline Intestinal & $64,0(30)$ & $63,0(37)$ & \\
\hline Difuso & $36,0(17)$ & $37,0(22)$ & \\
\hline Grado de Diferenciación (\%) & & & 0,10 \\
\hline Bien diferenciado & $36,0(17)$ & $19,0(11)$ & \\
\hline Moderadamente diferenciado & $26,0(12)$ & $39,0(23)$ & \\
\hline Poco diferenciado & $38,0(18)$ & $42,0(25)$ & \\
\hline
\end{tabular}



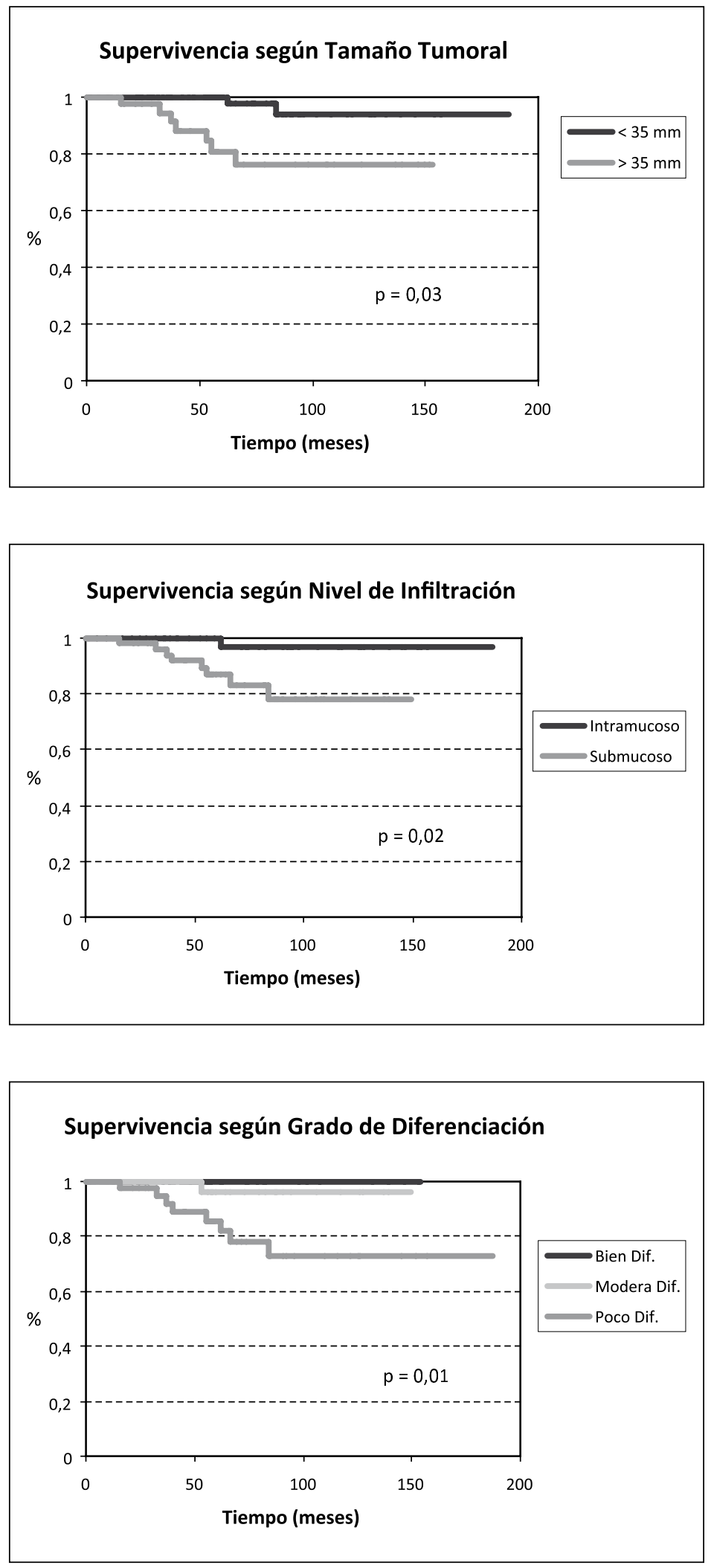

Figura 1. Supervivencia en pacientes con CGI según el diámetro tumoral mayor.

Figura 2. Supervivencia en pacientes con CGI según el nivel de infiltración tumoral en la pared gástrica.

Figura 3. Supervivencia en pacientes con CGI según el grado de diferenciación histológico. 
FACTORES MORFOLÓGICOS ASOCIADOS AL PRONÓSTICO DE PACIENTES OPERADOS POR CÁNCER GÁSTRICO...

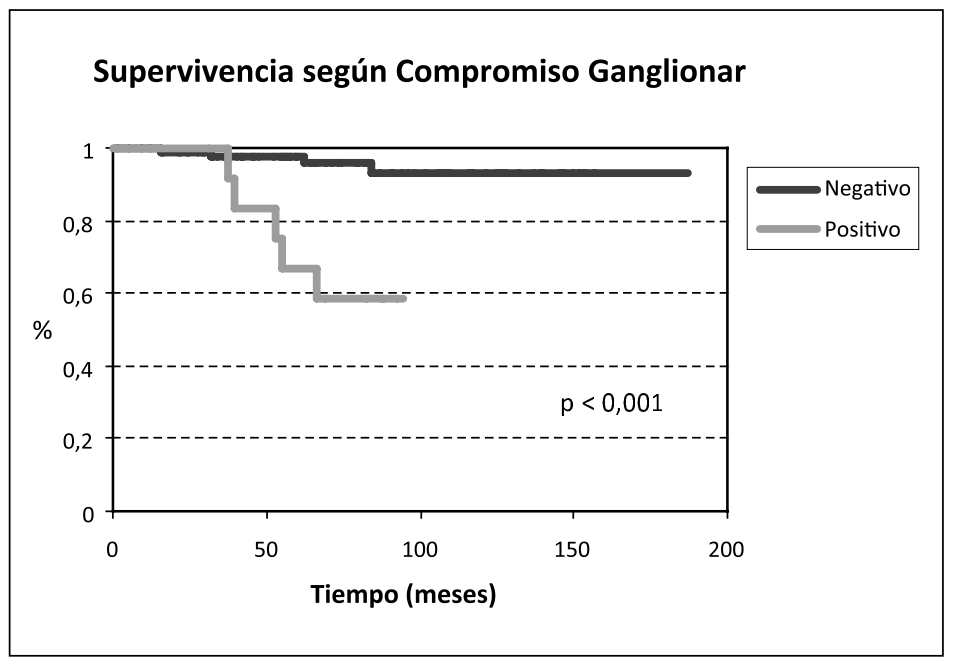

Figura 4. Supervivencia en pacientes con CGI según compromiso ganglionar linfático.
Tabla 2. Análisis multivariado

\begin{tabular}{lccl}
\hline Variable & OR & IC 95\% & p \\
\hline Tumor $\leq 35 \mathrm{~mm}$ & 6,6 & $1,3-31,9$ & 0,01 \\
Tumor submucoso & 7,9 & $0,9-63,6$ & 0,05 \\
Tumor difuso de Lauren & 7,9 & $1,8-28,5$ & 0,005 \\
Tumor poco diferenciado & 8,1 & $1,2-55,7$ & 0,03 \\
Compromiso ganglionar & 8,0 & $2,1-30,2$ & 0,002 \\
\hline
\end{tabular}

\section{Discusión}

Dada su frecuencia y altas tasas de mortalidad, el CG constituye en nuestro país un problema de salud pública, siendo generalmente estos tumores diagnosticados en etapas avanzadas con la consiguiente menor supervivencia y mayor morbilidad ${ }^{12-14}$. Dado lo anterior, hoy en día lo más importante parece ser pesquisar cada vez más tumores incipientes mediante la ejecución de programas eficaces de detección precoz que cuenten con médicos endoscopistas y anatomo-patólogos entrenados, ya que estos tumores presentan SV significativamente mayor que tumores avanzados ${ }^{5,7}$. Con esta finalidad, en nuestro país se han creado políticas de salud, vigentes desde julio de 2006, las que tienen por objetivo disminuir la mortalidad por CG, dando orientaciones enfocadas tanto a la prevención como identificación de población de riesgo, en los cuales la realización de una endoscopia digestiva alta permitiría aumentar la pesquisa de tumores gástricos en estadios tempranos junto con garantizar su tratamiento en tiempos establecidos ${ }^{6,12,15}$.
La prevalencia del CGI es ampliamente variable dependiendo de la población estudiada, así en Japón corresponde aproximadamente a un 50\% de los CG diagnosticados, mientras que estudios nacionales han reportado cifras que no superan el $20 \%$, correspondiendo en nuestra serie al $15 \%$ con una tasa de SV global a 5 y 10 años similares a la publicada por otros autores $(91 \% \text { y } 86 \%)^{2-6}$. En países con alta frecuencia de CG, como Japón y Corea, se aplican con éxito programas de tamizaje masivo, detectándose aproximadamente 1 caso por cada 800 pacientes examinados ${ }^{16-18}$. La experiencia a nivel nacional de estos programas data de 1978, reportándose en esos tiempos una tasa de detección de $0,43 \%$ en población general, mientras que en grupos sintomáticos esta alcanzaba al $1,27 \%$, correspondiendo a tumores incipientes el $14 \%$ y $11 \%$ de los casos para cada uno de estos grupos. Hoy en día, expertos nacionales, no consideran practicable tamizajes poblacionales masivos dado su escasa relación costo/efectividad y limitaciones logísticas; mientras que los resultados de programas de tamizaje selectivos ( $>40$ años y sintomáticos) son controversiales, existiendo trabajos con resultados promisorios que reportan pesquisas de aproximadamente 1 caso por cada 40 50 endoscopias y de los cuales un $20 \%$ son tumores incipientes $\mathbf{s}^{12,15,19,20}$.

Está ampliamente documentada la importancia pronóstica del compromiso ganglionar linfático para sujetos con CGI, con frecuencias reportadas de metástasis nodal entre 5\% y $20 \%$, observándose compromiso ganglionar hasta en el $3 \%$ de tumores intramucosos y $20 \%$ de submucosos ${ }^{4,-10}$. En nuestro grupo constatamos 13 casos (12\%) con metástasis ganglionar (4\% intramucoso y 19\% submucoso), 
confirmando su valor pronóstico en estos tumores $(\mathrm{p}<0,001)$ y demostrando también una relación directa del estado nodal con el tamaño tumoral $(\leq 35$ $\mathrm{mm} y>35 \mathrm{~mm})(\mathrm{p}=0,01)$, grado de diferenciación histológico $(\mathrm{p}=0,02)$, tipo histológico según Lauren $(\mathrm{p}=0,001)$ y nivel de infiltración parietal (intramucoso y submucoso) $(\mathrm{p}=0,02)$, lo que concuerda con lo previamente publicado, donde sus autores mencionan a estas variables como factores de riesgo independientes para metástasis ganglionar linfática en $\mathrm{CGI}^{4,5,8,21,22}$.

El análisis multivariado permitió verificar asociación con altos valores de OR, sin embargo, se ha de considerar que los intervalos de confianza son muy amplios y en el caso de "tumor submucoso" uno de sus extremos es inferior a 1; situación que es coincidente con un valor de $\mathrm{p}=0,05$; razón por la que no se le ha de considerar como un factor asociado a la SV de pacientes con CGI.

Así también demostramos que aquellos tumores localizados en el $1 / 3$ superior, $>35 \mathrm{~mm}$, poco diferenciados y/o infiltrantes en la túnica submucosa presentan SV significativamente más bajas; variables que como se menciona anteriormente presentan una relación directa con el estado ganglionar. En cuanto al valor pronóstico de la variable localización tumoral, debemos mencionar que si bien demostró en el grupo general ser un factor asociado al pronóstico; al analizar esta variable para cada uno de los grupos no observamos diferencias estadísticas significativas en la SV de tumores intramucosos $(\mathrm{p}=0,25)$ ni submucosos $(\mathrm{p}=0,09)$.

En este trabajo sólo se incluyeron pacientes sometidos a gastrectomía total o subtotal con disección ganglionar D2, no observando diferencias significativas en la SV según el tipo de cirugía realizada. Hoy en día se recomienda realizar procedimientos menos invasivos y resecciones más económicas en sujetos con CGI, ya sea por vía endoscópica o laparoscópica, de modo de obtener una mejor calidad de vida y reducir los costos quirúrgicos comparados con la vía abierta. La resección por vía laparoscópica es una técnica ya validada en Japón y Corea como alternativa de tratamiento curativo para CGI y ampliándose sus indicaciones a algunas lesiones avanzadas en la medida que se asegure primeramente su intención oncológica curativa ${ }^{4,7,8,23}$. Si bien, a nivel nacional la experiencia es escasa, los resultados postoperatorios inmediatos satisfactorios hacen de ella una atractiva alternativa a la cirugía abierta, sin embargo, es necesario todavía evaluar con estudios controlados a largo plazo sus resultados, compararlos con los tratamientos convencionales y de esta forma asegurar su criterio oncológico ${ }^{24-27}$. Por otro lado, resecciones por vía endoscópica, requieren disponer de equipamiento y endoscopistas entrenados, siendo una de las principales desventajas en las resecciones endoscópicas el no disponer de los ganglios linfáticos para examen histopatológico y por lo tanto no conocer el estado ganglionar $(\mathrm{N})$. Es importante mencionar como otro de los inconvenientes en las resecciones menos invasivas (mucosectomía y disección submucosa) la existencia de lesiones sincrónicas o multifocales en el 5 a $15 \%$ de los casos de CGI con recurrencias reportadas de hasta $13 \% 0^{4,28,29}$.

En base a lo anteriormente expuesto, podemos concluir que se verificó una prevalencia de CGI de $15 \%$, los que resecados presentan una SV de $91 \%$ a 5 años. El compromiso ganglionar linfático es un factor asociado a la SV; y además, se relaciona con el tamaño tumoral, grado de diferenciación histológico, tipo histológico según Lauren y nivel de infiltración parietal.

\section{Referencias}

1. Murakami T. Pathomorphological diagnosis. Definition and gross classification of early gastric câncer. Gann Monogr cancer Res. 1971;11:53-5.

2. Itoh H, Oohata $Y$, Nakamura K, Nagata T, Mibu R, Nakayama F. Complete ten-year postgastrectomy followup of early gastric cancer. Am J Surg. 1989;158:146.

3. Endo M, Habu H. Clinical studies of early gastric cancer. Hepatogastroenterology 1990;37:408-10.

4. Pelza J, Merkela S, Horbacha T, Papadopoulosb T, Hohenbergera W. Determination of nodal status and treatment in early gastric cancer. EJSO 2004;30:935-41.

5. Butte JM, Torres J, Viviani P, Duarte I, Crovaril F, Guzmán S, y cols. Sobrevida alejada de pacientes operados por cáncer gástrico incipiente. Rev Med Chile 2008;136:1424-30.

6. Guías clínicas GES 2006 para Cáncer Gástrico, Ministerio de salud-Chile.

7. Sano T, Hollowood A. Early gastric cancer: diagnosis and less invasive treatments. Scandinavian Journal of Surgery 2006;95:249-55.

8. Okabayashi T, Kobayashi M, Nishimori I, Sugimoto T, Namikawa T, Onishi S, et al. Clinicopathological features and medical management of early gastric cancer. The American Journal of Surgery 2008;195:229-32.

9. Popiela T, Kulig J, Kolodziejczyk P. Long-term results of surgery for early gastric cancer. Br J Surg. 2002;89: 1035-42.

10. Basili G, Nesi G, Barchielli A. Pathologic features and longterm results in early gastric cancer: report of 116 cases $8-13$ years after surgery. World J Surg. 2003;27:149-52.

11. Shimada S, Yagi Y, Shiomori K. Characterization of early gastric cancer and proposal of the optimal therapeutic strategy. Surgery 2001;129714-9.

12. Calvo A, Pruyas M, Nilsen E, Verdugo P. Frecuencia de 
FACTORES MORFOLÓGICOS ASOCIADOS AL PRONÓSTICO DE PACIENTES OPERADOS POR CÁNCER GÁSTRICO...

cáncer gástrico en endoscopias realizadas en pacientes sintomáticos en centro de atención secundario de salud. Rev Med Chile 2001;129:749-55.

13. Buonadonna A, Lombardi D, De Paoli A, Bidoli E, Frustaci S. Adenocarcinoma of the stomach: univariate and multivariate analyses of factors associated with survival. Suppl Tumori 2003;5:S31-4.

14. Green D, Ponce de León S, León-Rodríguez E, SosaSánchez R. Adenocarcinoma of the stomach: univariate and multivariate analysis of factors associated with survival. Am J Clin Oncol. 2002;25:84-9.

15. Hoffemberg P, Bahamondes G, López H, Cozzi V, Rufin F, Dávila M, y cols. Pesquisa endoscópica de lesiones gástricas en voluntarios aparentemente sanos. Rev Med Chile 1978;106:586-90.

16. Tsubono Y, Nishino Y, Tsuji I, Hisamichi S. Screening for Gastric Cancer in Miyagi, Japan: Evaluation with a Population-Based Cancer Registry. Asian Pac J Cancer Prev. 2000;1:57-60.

17. Mizoue T, Yoshimura T, Tokui N, Hoshiyama Y, Yatsuya H, Sakata K, et al. Japan Collaborative Cohort Study Group. Prospective study of screening for stomach cancer in Japan. Int J Cancer 2003;106:103-7.

18. Hisamichi S. Screening for gastric cancer. World J Surg. 1989;13:31-7.

19. Llorens P. Gastric Cancer Mass Survey in Chile. Semin Surg Oncol. 1991;7:339-43.

20. Llorens P. Resultado del examen masivo del cáncer gástrico en Chile, diagnóstico y tratamiento de las afecciones gástricas. Eds. Llorens P, Nakamura K. Instituto Chileno Japonés de Enfermedades Digestivas. Agencia de Cooperación Internacional del Japón (JICA). Tokio 1995: 143-60.
21. Nieminen A, Kokkola A, Ylä-Liedenpohja J, Louhimo J, Mustonen H, Puolakkainen P. Early gastric cancer: clinical characteristics and results of surgery. Dig Surg. 2009;26:378-83.

22. Wu ZM, Wu AW, Li ZY, Wu Q, Zhang LH, Wu XJ, et al. Characteristics of lymph node metastasis and prognostic analysis in 157 early gastric cancer patients. Zhonghua Wei Chang Wai Ke Za Zhi 2009;12:350-3.

23. Shiraishi N, Yasuda K, Kitano S. Laparoscopic gastrectomy with lymph node dissection for gastric cancer. Gastric Cancer 2006;9:167-76.

24. Escalona A, Báez S, Pimentel F, Calvo A, Boza C, Viñuela $\mathrm{E}$, y cols. Gastrectomía laparoscópica en cáncer gástrico. Rev Chil Cir. 2008;60:188-93.

25. Escalona A, Pérez G, Crovari F, Boza C, Pimentel F, Devaud N, y cols. Gastrectomía laparoscópica en cáncer gástrico: Experiencia preliminar. Rev Med Chile 2007;135:512-16.

26. Llorens P, Pisano R, Pisano R. Mucosectomía endoscópica en lesiones malignas gástricas incipientes. Rev Méd Chile 2000;128:969-76.

27. Llorens P, Navarrete C. Terapéutica endoscópica del cáncer gástrico incipiente, diagnóstico y tratamiento de las afecciones gástricas. Eds. Llorens P, Nakamura K. Instituto Chileno Japonés de Enfermedades Digestivas. Agencia de Cooperación Internacional del Japón (JICA). Tokio; 1995:123-7.

28. Marrano D, Viti G, Grigoni W, Marra A. Synchronous and metachronous cancer of the stomach. Eur J Surg Oncol. 1987;13:493-8.

29. Kodera Y, Yamamura Y, Torii A, Uesaka K, Hirai T, Yasui $\mathrm{K}$, et al. Incidence, diagnosis and significance of multiple gastric cancer. Br J Surg. 1995;82:1540-3. 